\title{
Le sacrement de pénitence ou la Confession sous le Régime français (suite)
}

\section{Valérien Roy}

Volume 16, numéro 3, décembre 1962

URI : https://id.erudit.org/iderudit/302214ar

DOI : https://doi.org/10.7202/302214ar

Aller au sommaire du numéro

Éditeur(s)

Institut d'histoire de l'Amérique française

ISSN

0035-2357 (imprimé)

1492-1383 (numérique)

Découvrir la revue

Citer cet article

Roy, V. (1962). Le sacrement de pénitence ou la Confession sous le Régime français (suite). Revue d'histoire de l'Amérique française, 16(3), 409-427. https://doi.org/10.7202/302214ar d'utilisation que vous pouvez consulter en ligne.

https://apropos.erudit.org/fr/usagers/politique-dutilisation/ 


\title{
LE SACREMENT DE PÉNITENCE OU LA CONFESSION SOUS LE RÉGIME FRANÇAIS*
}

\author{
CHAPITRE II
}

\section{LA DOCTRINE DU SACREMENT DE PÉNITENCE TELLE QU'ENSEIGNEE DANS \\ LES CATECHISMES ET LES AUTRES DOCUMENTS}

Il faut maintenant en venir à ce point capital. Les fidèles devaient recevoir une instruction claire et solide au sujet de leurs obligations de pénitents. Rituel, Catéchismes, Mandements et Synodes se complètent pour former ce corps de doctrine sûre, qu'il fallait faire connaître en chaire, au confessionnal, en classe et au foyer. Parcourons done ce tout organique, en exposant une dizaine de points qui engloberont tout ce qu'on enseignait alors sur la Confession, et que, même les petits enfants devaient apprendre, dès l'usage de raison.

\section{Le 3e commandement de l'Église.}

Dans le Catéchisme St-Vallier, le $3^{\text {e }}$ commandement de l'Eglise est ainsi formulé et commenté:

Tous tes péchés confesseras,

à tout le moins une fois l'an. ${ }^{43}$

«L'Église, comme une bonne mère, écrit Mgr de St-Vallier, ne se contente pas de nous porter à la pratique de toutes les vertus par l'institution de fêtes; elle veut encore que nous nous purifiions de toutes nos iniquités, de peur que par dureté et impénitence de notre cœur, nous n'amassions un trésor de colère pour le jour de la vengeance du Seigneur, et que nous ne mourions pas dans nos péchés. $\gg^{44}$

* Voir notre Revue, XVI: 225-239.

43 Catéchisme St-Vallier, 211.

44 Ibid., 211s. 
L'obligation de la confession annuelle existait donc, comme aujourd'hui; et lo Catéchisme insiste sur ce fait que c'est bien Notre-Seigneur lui-même qui pose cette obligation de nous confesser; l'Eglise n'a déterminé que le temps de notre confession: au minimum, une fois l'an. ${ }^{4 \overline{5}}$ "Mais, comme le juste pèche 7 fois le jour, et qu'on paraît devant Dieu à l'heure où on $y$ pense le moins, on doit toujours avoir de l'huile dans sa lampe, de peur que l'arrivée de l'Epoux ne nous surprenne.» ${ }^{46}$ C'est pourquoi la Leçon précise bien qu'il est utile de multiplier ces confessions, en se présentant au moins une fois par mois et avant toutes les bonnes fêtes. ${ }^{47}$ Comme conclusion, l'auteur cite quelques exemples pour en illustrer le thème et en souligner la portée. $^{48}$ Le besoin d'une confession dite fréquente est souvent remis en question; ce qui nous montre bien que, du temps de Mgr de St-Vallier, le confessionnal ne devait pas être regardé comme un épouvantail ${ }^{+9}$.

\section{A qui se confesser : juridiction; billet de confession;} passeport; cas réservés.

a) Juridiction: Le pénitent ne peut généralement s'adresser qu'au seul Curé de sa paroisse. Dans le cas où il sentirait le besoin de s'adresser à un autre confesseur, il lui faudrait l'autorisation de son curé. Le Rituel ajoute que si un confesseur s'aperçoit qu'un pénitent vient à lui, de l'extérieur, toujours sans cause légitime, pour éluder son pasteur, il devra le renvoyer. ${ }^{50}$ Cependant, pour favoriser la liberté des consciences, le curé demandera de temps en temps à l'Évêque des confesseurs auxiliaires, mais pas n'importe qui, parce qu'il s'en trouve qui ne peuvent pas confesser dans les grandes paroisses, surtout les personnes inconnues. ${ }^{51}$ Il est entendu, d'ailleurs, que le curé n'a juridiction que dans sa paroisse, bien qu'il puisse recevoir en

45 Ibid., 212.

46 Ibid., 213.

47 Loc. cit.

48 Ibid., 213s. et 270s.

49 Gosselin, Vie de Mgr de St-Vallier, I: 33. Voir aussi, Mandements, I: 60,332 .

50 Rituel, 1 ère éd., 89.

51 Loc. cit. 
confession tous ceux qui se présentent, hors le temps pascal,52 car cette confession annuelle, obligatoire dans la quinzaine de Pâques, ${ }^{53}$ doit être faite dans sa propre paroisse. Les Vicaires généraux du diocèse de Québec répondant à une consultation au sujet de la confession pascale, spécifiaient que cette confession est non valable si le pénitent, sans permission, est allé à un confesseur en dehors de sa paroisse, sans être allé, auparavant ou après, une fois, à son Pasteur, durant ce même temps. ${ }^{54}$ Evidemment, ce n'est que par voie de conséquence que la juridiction concernait le pénitent.

A la lecture de quelques Mandements, on se rend compte que ce point disciplinaire de la juridiction inquiétait $\mathrm{Mgr}$ de St-Vallier. S'il apprend, par exemple, que plusieurs continuent de confesser sans faire renouveler leur mandat, il en est grandement étonné.55 Il rappelle les règles du Synode, que tous devaient savoir, et précise que le permis de confesser est de 3 ans pour les confesseurs des villes, séculiers et réguliers; quant aux missionnaires, ils obtiennent un permis valable pour 5 ans. Dans

52 Ibid., 88.

53 « Tous les Curés auront soin de connaître par eux-mêmes dans le temps de Pâques leurs Paroissiens, déclarant que la Confession et la Communion qui sera faite à un Missionnaire étranger ne sera point regardée comme une Communion Pascale. 》Statuts publiés dans le 1er Synode tenu à Québec, le 9 nov. 1690. Mandements, I: 271. - Le Concile de Trente (Session XIV, c. V) reprenait la doctrine du Concile de Latran et prescrivait la confession annuelle en carême. Vacant-Mangenot, à l'art. Confession, dans le Dictionnaire de théologie catholique, col. 906s.

54 Résolutions de quelques cas de conscience, dans Mandements, I:472477. - L'expression proprius sacerdos (à leur propre curé) a eu un sens longtemps discuté. Quelques théologiens n'y voyaient que le curé de la paroisse; ainsi, la plupart des théologiens du $13 \mathrm{e}$ et $14 \mathrm{e}$ siècles, rigoristes ou autres, qui tenaient à cette opinion, exigeaient qu'une autorisation de recourir à un autre confesseur pour la confession annuelle, fût sinon obtenue, du moins demandée au curé de paroisse. Dans les siècles qui suivent, cette opinion tombe de plus en plus en désuétude. Vacant-Mangenot, op. cit., col. 828-975.

$55 \ll .$. Nous ne saurions assez vous exprimer la douleur que nous avons conçue, en apprenant l'infraction manifeste des statuts de notre dernier Synode, particulièrement en ce qui regarde le tribunal de la pénitence. (...) Plusieurs ont la témérité de confesser encore depuis 13 années, sans avoir fait renouveler leur approbation. Quel dérèglement! Quel malheur ! Et quel en doit être le châtiment?...» Suivent des ordonnances sévères, et surtout cette mesure extrême: «... Nous révoquons tous les pouvoirs par nous donnés à nos dits Vicaires généraux, jusqu'à présent...» Mandements, de Paris, 1713, I: 482-486. 
la Vie de Mgr de Laval, on rapporte un scandale à ce sujet: un certain Père Louis, récollet, soutenu par son Provincial, exerce son ministère à Chambly, sans permission de l'Évêque. ${ }^{56}$ Ces mêmes Récollets avaient ouvert, à la haute-ville, une infirmerie qui se compléta bientôt d'une chapelle, où, contre le gré de Mgr de Laval, on faisait du ministère paroissial..$^{57}$ Il n'en fallait pas tant pour s'attirer les foudres du rude Prélat!

b) Billet de confession: ${ }^{58}$ Le billet de confession est un certificat exigé, originellement, de ceux qui se présentent pour contracter mariage, à moins que le célébrant lui-même ne les ait confessés. Il est requis afin de constater que ceux, pour l'enterrement desquels l'office de l'Église est demandé, se confessaient ordinairement à des prêtres acceptant sans réserves la Bulle Unigenitus et toutes les décisions du Saint-Siège s'y rapportant. ${ }^{59}$ Voici la formule ordinaire de ces billets: Audivi pro sacro tribunali $N$. In cujus rei fidem subscripsi, die... mensis ... anni... J'ai entendu en confession N. de la paroisse de N. En foi de quoi je lui ai délivré le présent certificat, à... le... du mois de... de l'année... Dans un vieux document, on rencontre cette autre formule: Je soussigné, prêtre missionnaire de Camourasca confesse avoir receu le Sieur Janeau au sacrement de penitence et luy ay donné la sainte communion. A Camourasca, ce $11^{e}$ Avril 1714. Auclair ptre $M e{ }^{60}$ Peu à peu, l'usage de ces billets se généralisa, surtout dans les Couvents, les Monastères et les Collèges, où l'autorité l'exigeait des inférieurs, comme moyen de contrôler la fréquence des confessions et des communions.

${ }^{56}$ Gosselin, Vie de Mgr de Laval, 92ss.

57 Ibid., 96 ss.

58 Ces billets prirent une place importante dans l'histoire du jansénisme; ils provoquèrent de grands conflits entre la Cour, les Evêques et le Parlement. Sous Charles X (1824-1830), les fonctionnaires qui ne se confessaient pas étaient menacés de perdre leur emploi. La Grande Encyclopédie, etc., à l'art. Billet, 866.

59 Loc. cit. - Le Père Bouvart parle longuement des Billets de confession dans sa critique du Rituel et du Catéchisme de Mgr de St-Vallier. Ce critique et son interlocuteur donnent leur avis sur la coutume. On peut voir un peu comment cet usage fonctionnait alors; c'était compliqué, et les deux théologiens s'en plaignent, en faisant allusion au système des billets établi au Collège des Jésuites. Gosselin, Le Rituel de Mgr de StVallier, dans MSRC, déc. 1914, 255ss.

60 André Vachon, Le notaire en Nouvelle-France, dans RUL, nov. 1955, où il cite APQ, Coll. de pièces jud. et not., no 2040. 
Procédé qui frisait l'indiscrétion, pour ne pas dire plus, car il prenait facilement l'allure d'une ingérence indue dans le for interne des sujets. Mais, c'était la mode ! et on la subissait quand on ne pouvait pas l'accepter de bon gré. Sans voir du jansénisme dans cette coutume, on y décèle bien cette rigidité méticuleuse qui entourait la réception des sacrements.

Or, la question des billets de confession revient ici ou là dans les documents; mais on remarque que les réglementations à ce sujet s'appuient sur une coutume déjà en vigueur au pays, plutôt que sur des normes établies par le Rituel ou les Catéchismes. Ces sources, en effet, se contentent de préciser que les Curés doivent, pour se conformer aux prescriptions du Concile de Latran, donner facilement ces billets pour se confesser à d'autres personnes, afin que les pénitents aient le choix entre plusieurs confesseurs. ${ }^{61}$ Bien plus, les Vicaires généraux consultés soutiennent que les confesseurs, en donnant des billets à leurs pénitents, par lesquels ils certifient qu'ils les ont entendus en confession, ne pèchent pas mortellement ni ne révèlent le secret sacramental, et cela, affirment-ils, en vertu de la coutume. ${ }^{62}$

Dans l'Histoire de la paroisse de Champlain ${ }^{63}$, on lit à ce propos une curieuse affaire, qu'une lettre du curé concerné ne rend pas suffisamment claire. Il s'agit de l'abbé François Morisseau, curé de Champlain, qui sent le besoin de se disculper

${ }^{61}$ Statuts du 1 er Synode tenu à Québec, le 9 nov. 1690, dans Mandements, I: 271.

$62 \mathrm{Ils}$ argumentent comme ceci: «Monseigneur l'évêque (St-Vallier) recommande aux confesseurs d'accorder sans difficultés ces sortes de billets, comme n'étant pas capables de faire tort au secret inviolable de la confession, et étant en usage dans les diocèses les mieux réglés du royaume. Saint Charles (Borromée) les prescrit, et ils sont d'ailleurs imprimés et donnés au public par la délibération des Evêques assemblés en France avec tout l'éloge et la recommandation possible. Ce cas ayant été proposé autrefois en Sorbonne, en 1674, il fut décidé que cet usage, surtout dans la quinzaine de Pâques, n'a rien qui soit contraire au secret et au sceau de la confession, pourvu que les billets soient uniformes et donnés indifféremment à tous ceux que les Curés respectifs ont entendus en confession. Celui qui soutiendrait une thèse contraire serait lui-même blâmable de condamner de la sorte un usage qui est approuvé des plus grands hommes, et reçu dans un grand nombre de diocèses.» Donné le 27 juin 1709. Chs Grandelet, Vic. gén., Louis Ango Des Maizerets, Vic. gén., J. Lacolombière, Vic. gén. Résolutions de quelques cas de conscience, dans Mandements, I: 480.

63 Cloutier, II: 153. 
auprès de son évêque, Mgr de Pontbriand. En substance, il dit qu'un certain Père Augustin doit faire des plaintes sur son compte, touchant ceux qui, des Trois-Rivières, ont l'habitude de venir à confesse à lui et qu'il a absous et à qui il a donné des billets. "Ce bon Père, précise le Curé, les a renvoyés disant que les confessions et les communions étaient sacrilèges, parce que faites en temps de Pâques, et qu'il n'y avait qu'eux (les Pères) qui avaient pouvoir d'absoudre les cas réservés... Il y a aussi de mes paroissiens, continue l'abbé Morisseau, qui ne se sont aucunement présentés dans le temps de Pâques, disant qu'au cours de l'été ils iront vous demander des billets et que vous ne les refuserez pas, ou bien qu'ils surprendront dans les pèlerinages, les curés voisins qui leur feront faire leurs Pâques. J'espère que votre Grandeur voudra tenir la main à tous ces désordres. »

Cette question des billets de confession semble avoir été parfois, et même souvent, cause de malaise et de chicane; une petite contrebande, si l'on peut utiliser ce terme, aurait été le fait de quelques confesseurs trop zélés... ou trop faibles !

c) Passeport: Les voyageurs qui sortent de leur territoire, doivent se munir d'une Lettre testimoniale qui attestera de leur statut de religieux et leur assurera, au besoin, les secours de la religion. En voici un modèle tiré textuellement du Rituel StVallier:

Prêtre curé de la Paroisse de N. Diocèse de N. A tous ceux qui ces présentes lettres verront, Salut. Je certifie que N. mon Paroissien n'est lié d'aucune Censure Ecclésiastique, ny affecte d'aucune heresie ou mauvaise doctrine, qu'au contraire il est de bonne reputation, de vie exemplaire, \& fidèle observateur de la Religion Catholique, Apostolique \& Romaine. C'est pourquoi, si en allant ou revenant, il a besoin des secours tant spirituels que corporels, des Sacrements, \& de la Sépulture, nous vous supplions, de ne pas les luy refuser. En foy de quoy j'ay signé de ma propre main les presentes ce du mois de mil 64

64 Rituel, Ière éd., 561. 
Si le voyageur doit sortir du Royaume, il faut luy donner l'attestation en Latin. ${ }^{65}$

d) Cas réservés: La question de juridiction pour entendre les confessions pose celui des cas réservés. On avait tendance à les multiplier; Mgr de St-Vallier, tout spécialement, tient à rappeler souvent aux confesseurs les règles fixées en la matière. Voyons les principaux cas pour l'absolution desquels l'Évêque se réservait la juridiction ${ }^{66}$ :

1. Dans le cas de ceux qui avec scandale profèrent, font ou écrivent quelque chose extraordinairement injurieux contre Dieu, la Sainte-Vierge, les Saints, et les choses sacrées.

2. Cas de celui qui frappe son père ou sa mère et leur refuse le secours jugé nécessaire.

3. Cas de ceux qui exercent la magie ou de ceux qui les consultent.

4. Dans le cas d'inceste avec parent au 1er et $2 \mathrm{e}$ degré.

5. Cas de sodomie et de bestialité.

6. Cas d'adultère ou de concubinage public, et cas de viol.

7. Cas de celui qui mange de la viande durant le carême ou les jours défendus.

8. Cas de ceux qui vont à la chasse à l'Orignal pendant le Carême, sans la permission de leur curé.

9. Dans le cas de libelles et de chansons diffamatoires.

10. Dans le cas de duel.

11. Cas de celui qui ne paie pas la dîme dans le temps pascal.

12. Dans le cas d'impureté commise par les Français avec les femmes ou les filles sauvages.

13. Dans le cas de sacrilège, si on communie en état de péché mortel.

Mandement pour les cas réservés, dans Mandements, 
14. Contre celui qui enivre les sauvages, leur donne ou vend des boissons assez pour les enivrer, à moins d'assurance du contraire ${ }^{67}$.

Les cas réservés au Pape sont moins nombreux, mais ils existent et sont énumérés comme suit, dans le Rituel ${ }^{68}$ :

1. Cas de simonie.

2. Cas de confidence commis par celui qui reçoit ou retient un bénéfice.

3. Cas de celui qui brûle ou pille une église, un monastère, si ce crime est dénoncé publiquement.

4. Cas de l'incendie d'un lieu sacré, si l'auteur est excommunié.

5. Cas de falsification des Lettres apostoliques et des Bulles, etc.

6. Cas de celui qui maltraite un Clerc, si celui-ci meurt des coups reçus, ou s'il est mutilé.

7. Cas de la profanation religieuse dans le lieu saint.

Il est à noter que l'Évêque peut absoudre tous ces cas s'il sont occultes, sauf dans celui d'homicide; ou encore, ces cas peuvent être absous en faveur de ceux que le Droit exempte d'aller à Rome: religieuse, fille, vieillard, etc. ${ }^{69}$

\section{Examen de conscience. Occasion prochaine.}

A la fin du Rituel, figure une Table complète des points pouvant servir à l'examen de conscience avant la confession. Il

67 « Nous nous réservons le péché qu'il y a à enivrer les sauvages et à leur vendre ou donner des boissons à transporter en quantité suffisante pour les enivrer, à moins qu'il y ait une assurance morale qu'eux ou d'autres sauvages ne s'en enivreront pas. Comme aussi de ceux qui abusent des filles ou femmes sauvages devant ou après la boisson ou en quelque manière que ce soit. 》 Cas réservés, 21 avril 1669, Mandements, I: 77. — Les discussions à ce sujet ont rempli une bonne partie de l'épiscopat de Mgr de Laval. Les maîtres en Sorbonne se prononcèrent sur ce pouvoir que s'arrogeait l'évêque, et donnèrent raison à $\mathrm{Mgr}$ de Laval, disant qu'il peut en faire un cas réservé. Le 8 mars 1675 , Mandements, I: $91-94$; 149-156; 328-331. Voir aussi, Rituel, 1ère éd., 105ss. - Dans Résolution de quelques cas de conscience, " on demande si les confesseurs qui n'ont pas reçu de l'Evêque ou des Grands Vicaires, le pouvoir d'absoudre des cas réservés à l'Evêque, le peuvent faire en conscience, et validement? - Résolution: Il est constant qu'ils ne le peuvent, et l'opinion contraire a été condamnée par le Pape Alexandre VII, comme étant fausse, téméraire, scandaleuse, erronée et tendant à l'hérésie et au schisme. 》 Mandements, I: 477. 68 Rituel, 1 ère éd., 104s.

69 Loc. cit. 
est ordonné aux curés d'expliquer cet examen à leurs paroissiens, le premier dimanche de Carême de chaque année, afin de les mieux disposer à faire une bonne confession pascale. ${ }^{70}$

Le pénitent est exhorté à s'examiner sérieusement sur cet item capital de l'occasion prochaine dans laquelle il peut se trouver; s'il ne veut pas en sortir, qu'il sache que sa résistance pourrait amener de la part du confesseur un refus d'absolution, surtout s'il s'agit d'impureté.71

\section{Manière de se confesser.}

Cette façon est sensiblement celle que nous utilisons encore de nos jours. Le pénitent, à genoux, tourné vers le fond du confessionnal, fait le signe de la croix, puis commence: Mon père, bénissez-moi, parce que j'ai péché. Il récite ensuite le Confiteor en français ou en latin, ${ }^{72}$ jusqu'au mea culpa. Il dit depuis combien de temps il est allé à confesse; puis vient l'accusation proprement dite des péchés. Il est bon qu'il prie ensuite le confesseur de l'interroger. Il termine le Confiteor, écoute les avis du confesseur, récite l'acte de contrition, puis écoute la pénitence donnée. Après avoir remercié le confesseur, il se retire et fait au plus tôt la pénitence sacramentelle. ${ }^{73}$

\section{La confession générale.}

On connaissait bien alors la pratique de la confession générale. Le Rituel la recommande fortement, explique les cas où elle est nécessaire, conseille de la faire surtout avant le choix

70 Ibid., 605s.

71 Ibid., 96-101.

72 Voici, à titre de curiosité, le Confiteor, tel qu'il était alors formulé: « Je me confesse à Dieu tout-puissant, à la bienheureuse Marie toujours Vierge, au bienheureux saint Michel Archange, au bienheureux saint JeanBaptiste, aux Saints Apôtres Pierre et Paul, à tous les Saints et à vous, mon Père, de tous péchés que j'ai commis par pensées, par paroles, et par mes actions. J'en dis ma coulpe, ma coulpe, ma très-grande coulpe, et partant je prie la bienheureuse Vierge Marie toujours Vierge, le bienheureux Saint Michel Archange, le bienheureux saint Jean-Baptiste, les SS. Apôtres Pierre et Paul, et tous les Saints et vous, mon Père, d'intercéder pour moi auprès du Seigneur Nôtre Dieu. \$ Rituel, 1ère éd., 143.

73 Catéchisme St-Vallier, 280ss. 
d'un état de vie, et expose une méthode de s'en acquitter avec le plus de fruits possible. ${ }^{74}$

6. De la contrition; ses qualités; les sortes de contrition; les marques de la contrition; diverses formules d'acte de contrition.

Il est assez évident que ce sont autant de points fortement soulignés, puisque, en définitive, la confession trouve en ce sentiment de douleur de ses péchés toute sa puissance de résurrection spirituelle et de paix. Les formules de l'acte de contrition prenaient diverses formes; en voici une: "Mon Dieu, je vous demande pardon de tous les péchés que j'ai commis en cette journée, \& en toute ma vie, je m'en repens de tout mon cœur, pour l'amour que je vous porte, \& je me resous d'en faire pénitence, \& de n'y plus retomber à l'avenir, moyennement vôtre sainte grâce, que je vous demande de tout mon cour. $\gg^{\tau 5}$

A la leçon IXe, le Catéchisme détaille avec précision cette partie majeure du sacrement de Pénitence; et, tout comme dans le Rituel, il signale la distinction très importante à établir entre la contrition parfaite et la contrition imparfaite. Rien qui sente le jansénisme dans cet enseignement; l'explication est constamment appuyée sur des textes de la Sainte Écriture et sur des exemples tirés de la même source inspirée. ${ }^{76}$ Nous croyons inutile d'entrer dans toutes les particularités d'une théorie encore si courante, et qui prouve qu'en dépit d'un rigorisme de fait, la doctrine alors communément enseignée sur ce point capital de la Pénitence restait parfaitement orthodoxe.

74 Rituel, 1ère éd., 102s.

75 Les formules de l'Acte de contrition ont peu varié depuis; en voici deux que donne le Catéchisme St-Vallier, à différentes pages: "Mon Dieu je suis marri de tout mon cœur de vous avoir offensé, parce que vous êtes infiniment bon, et que le péché vous déplaît; je fais une ferme résolution de m'en corriger à l'avenir moyennement vôtre sainte grâce, et de m'en confesser au plus tôt. 》 Ibid., 466. - «Mon Dieu je suis marri de vous avoir offensé, parce que vous êtes infiniment bon et que le péché vous déplaît, je propose de plutôt mourir que de vous offensé à l'avenir; je vous supplie par les mérites de votre Fils, mon Sauveur, de m'accorder la rémission de mes péchés et la grâce d'en faire pénitence tout le cours de ma vie. 》 Ibid., 508.

76 Ibid., 271ss. 


\section{La Satisfaction: pénitences imposées. Les Indulgences.}

La satisfaction sacramentelle consistera d'abord et surtout dans l'accomplissement de la pénitence imposée par le confesseur, qui se doit d'en donner une, et de la proportionner aux péchés accusés. ${ }^{77}$ Les pénitences données en confession étaient généralement plus coûteuses que celles imposées aujourd'hui pour des fautes de même gravité. Il sera intéressant de jeter un coup d'œil sur les exemples que suggère le Rituel, ou que rapporte la petite histoire, pour les fautes publiques.

Le confesseur doit étudier son pénitent avant de lui donner une pénitence. A titre de suggestions, voici ce qu'il pourrait imposer, selon la nature des péchés et selon leur gravité ${ }^{78}$ :

- D'abord, éviter les lieux, occasions, compagnies, danses qui ont été cause plus ou moins directe des fautes.

- Visiter les malades, le Saint-Sacrement. Faire de nombreuses génuflexions, des actes d'amour de Dieu. S'appliquer à son devoir d'état. Assister aux instructions à l'église. Réciter la prière du matin et du soir, à genoux. Faire quelques lectures spirituelles. Jeûner durant quelques jours. Faire des aumônes.

- Imposer le silence de quelques jours au blasphémateur. Lui ordonner de ne pas s'excuser devant de fausses accusations. Réciter des prières, prosterné la face contre terre. Baiser la terre. Réciter la prière en famille.

- Si quelqu'un manque la messe, il fera une aumône selon la grosseur de son gain habituel, ou de ses revenus en argent ou en nature. Il assistera à tous les offices du dimanche et des fêtes.

- S'il ignore la doctrine, il devra assister aux Instructions qui se donnent à l'église pour les gens et les enfants.

- L'impudique devra jeûner, s'abstenir de vin, coucher sur la dure, porter le cilice ou une ceinture de corde ou de fer, etc.

- L'ivrogne et le gourmand devront éviter les festins et se priver de vin, etc.

77 Rituel, 1 ère éd., 113.

78 Ibid., 113ss. 
- L'usurier fera restitution des biens mal acquis, et même compensera ses fraudes par des aumônes proportionnées. ${ }^{79}$

Mgr de St-Vallier rappelle que le confesseur doit être sévère comme ceux de la primitive Église, sans cependant dépasser les bornes. ${ }^{80}$

On rapporte qu'en 1773 un certain Sieur Camanne, voulant se réconcilier avec l'Église, Mgr J.-O. Briand, alors évêque de Québec, intervint et prescrivit au curé du converti, l'abbé Jean Baptiste Gatien, de Ste-Croix, le genre de pénitence qu'il devait imposer à son pénitent: "Vous lui donnerés (...) pendant un an de rester à cette même place (sous la cloche) toutes les fêtes et dimanches, à messe et à vêpres, et le condamnerés au pain et à l'eau tous les vendredis de l'année, et à l'obligation de réciter tous les jours à genoux, 5 Pater et Ave. $»^{81}$

Cette pénitence ressemble étrangement aux châtiments que pouvaient imposer, en ce temps-là, les autorités civiles. Ainsi, en 1733, Pierre Sillon dit Larochelle et Geneviève Millet vivaient ensemble, non mariés. Or l'Intendant, informé du fait, les condamne à faire amende honorable en chemise, la corde au cou, tenant en main une torche de cire ardente de deux livres, devant la principale porte de l'église cathédrale, tête nue et à genoux, et de déclarer à haute et intelligible voix que méchamment et frauduleusement ils ont profané et abusé de la sainteté du mariage, qu'ils se repentent et demandent pardon à Dieu. Ensuite ils seront battus et fustigés de verges par l'exécuteur de la haute justice, par les carrefours et les lieux accoutumés de cette ville (Québec) et bannis pour trois ans. ${ }^{82}$

${ }^{79} \mathrm{Mgr}$ de St-Vallier fait une longue dissertation sur l'usure et prévoit la peine d'excommunication comme possible. 22 déc. 1697. Mandements, I: 363ss. - Les Statuts du Synode de 1694 ordonnent de refuser l'absolution aux usuriers. Ibid., 316-322.

80 Rituel, 1ère éd., 115.

81 Inventaire de la Correspondance de Mgr Jean-O. Briand, évêque de Québec. (1741-1791), par Ivanhoë Caron, dans Rapport de l'Archiviste de la Province de Québec, pour l'année 1929-1930, 101. (Abrégé: RAPQ)

82 Gosselin, L'Eglise du Canada, V: 274s. - Voir: Crimes et peines sous le régime français. Comment on punissait les calomniateurs sous le régime français, dans Roy, Les petites choses de notre histoire, III: 197200. 
Le confesseur ne doit jamais imposer ce genre de pénitence publique pour des fautes secrètes, et, au cas où il jugerait utile d'infliger une pénitence publique avec port du cierge, etc., jamais il ne devra le faire sans le sentiment de l'Évêque. ${ }^{83}$

Comme moyen de réparer sans trop de frais la peine due aux péchés, la pratique des Indulgences était reconnue et enseignée dans les Catéchismes, le Rituel et les prônes. ${ }^{84}$ Toutefois, comme pour mettre en garde contre des abus possibles, le Catéchisme demande: "Doit-on se tenir en sûreté, après avoir tâché de gagner des indulgences ?»- Réponse: «Non ... on doit toujours travailler à son salut avec crainte et tremblement.» Ensuite, l'auteur tente d'expliquer ce qu'on entend par une indulgence de 7 ans et 7 quarantaines, en disant tout bonnement que par cette indulgence on obtient autant de valeur expiatrice que par 7 ans de pénitence ${ }^{85}$.

\section{Refus d'absolution.}

A lire les Mandements des Évêques et même les simples Catéchismes, il est hors de doute que le refus de l'absolution au pénitent était, ce qu'on pourrait appeler, une coutume sacramentale, en ce sens qu'on devait renvoyer le pénitent ou refuser de le recevoir, dans une foule de cas que détaillent minutieusement le Rituel et les Ordonnances épiscopales. Ainsi, dans le Synode tenu à Ville-Marie, le 8 mars 1694, les membres réunis sous l'autorité de l'Évêque dressent la liste des cas où l'absolution doit être refusée, ou différée. En cela on ne fait que rééditer une Ordonnance de Mgr le Cardinal de Grimaldy, archevêque d'Aix, déjà reçue et autorisée pour le Diocèse de Québec. ${ }^{86}$ Souvent ces cas sont donnés sans commentaire; de toute évidence, le confesseur devait tenir compte des circonstances avant de les appliquer; ici, une simple énumération suffira. On refusera l'absolution à celui :

${ }^{83}$ Rituel, 1ère éd., 117.

84 Catéchisme St-Vallier, 283-286; Rituel, 1ère éd., 119.

85 Catéchisme St-Vallier, 284s.

86 Mandements, I: 322-325; Rituel, 1ère éd., 96; appendice au Rituel, 1 1ère éd., 50-53. 
1. qui ne manifeste pas de marques de douleur de ses péchés.

2. qui ne veut pas restituer ou réparer le tort causé.

3. qui entretient une inimitié avec son prochain, sans vouloir pardonner.

4. qui ne quitte pas l'occasion prochaine.

5. qui met les autres dans l'occasion de pécher, comme, par exemple:

s'il tient une Assemblée impie;

s'il donne des représentations lascives ou exibe des tableaux immoraux;

s'il compose, édite ou vend des mauvais livres ;

s'il tient dans un métier où il est moralement impossible de ne pas offenser Dieu ;

si les femmes et les filles vont les seins découverts.

6. qui n'est pas instruit de la foi.

7. qui est reconnu pécheur public.

8. qui, ecclésiastique, laisse la soutane ou la résidence.

9. qui, ecclésiastique, prête de l'argent à intérêt.

10. qui, chirurgien ou barbier, fait le poil le dimanche et fêtes, ou se livre à des œuvres serviles.

Mgr Languet, dans son Instruction pour préparer à la première communion, réduit ces cas à six ${ }^{87}$ : refus d'absolution:

1. si l'on ignore le catéchisme,

2. si l'on garde une inimitié et que l'on ne se réconcilie pas,

3. si l'on retient le bien d'autrui sans vouloir restituer,

4. si, ayant blessé la réputation du prochain, on ne veut pas réparer,

5. si l'on reste dans l'habitude du péché et que l'on ne travaille pas à se corriger,

6. si l'on reste dans l'occasion du péché et ne s'en retire pas. 
D'où l'on voit que les enfants apprenaient, tout jeunes, que l'absolution pouvait leur être refusée, qu'elle était précieuse et qu'il fallait la mériter.

Comme il est rappelé dans un Mandement, les confesseurs ne sauraient être trop exacts à refuser l'absolution à tous ceux qui se rendent coupables de ces fautes et s'entêtent à demeurer dans un état incompatible avec l'amour du bon Dieu. Il faudra la refuser surtout à ceux qui forment des inimitiés ou des jalousies par leurs médisances, et qui vont à anéantir la charité envers leurs frères. ${ }^{88}$ Que les confesseurs sachent également différer l'absolution à ceux qui disent que ceux qui s'abstiennent de communier font ainsi parce qu'ils n'ont pas reçu l'absolution; ça les guérira. ${ }^{89}$

Mgr de St-Vallier tonne souvent devant les abus qui créent des scandales et dont se rendent coupables les femmes et les filles qui s'affublent de mises immodestes, surtout à l'église. «Il ne faut pas recevoir ces personnes aux Sacrements, au pain bénit, à l'offrande, aux quêtes. ${ }^{90}$ Quant à ceux qui abusent des loisirs du dimanche pour faire des voyages, pour vendre leurs produits, etc., on leur refusera l'absolution. ${ }^{91}$ On punira du même refus ceux qui sortent du prône plusieurs fois, car «c'est la plus grande marque d'irréligion qu'on puisse donner ${ }^{92}$ Dans une Ordonnance du 10 février 1732, le Grand vicaire Chartier de Lotbinière défend de porter Camail durant la messe et de sortir durant la prédication, à peine d'être refusé aux Sacrements. ${ }^{93}$ D'ailleurs, sur ce point, l'autorité civile elle-même doit veiller et imposer des sanctions: elle oblige à une amende de 10 livres ceux qui sortent pendant le Prône, qui se querellent ou conversent durant les messes, ou qui fument à la porte de l'église durant ce temps. ${ }^{94}$ En les refusant à la confession, on essaiera de guérir les blasphémateurs, ceux qui, sans manifester le désir de se corriger, disent des paroles déshonnêtes ou à

88 Mandements, I: $316-322$.

89 Ibid., 320.

90 Ibid., $106 \mathrm{ss}$, et Gosselin, Vie de Mgr de Laval, II : 319.

91 Mandements, I : 262.

92 Ibid., 361, 540s.

93 Ibid., 540.

94 Edits et Ordonnances, II : 425. 
double sens ${ }^{95}$. De même, ne sera pas absous celui qui ne paie pas ses dîmes ou qui ne les aura pas payées fidèlement ${ }^{96}$. Contre cette mesure, le comte Jérôme de Pontchartrain écrit à Mgr de St-Vallier, le 6 mai 1702, pour lui dire que ce refus est considéré comme trop rigoureux par sa Majesté. Pontchartrain est d'avis qu'on pourrait engager les habitants à payer une demie de la dîme à Pâques, et à en remettre l'autre moitié à un marguillier ou principal habitant pour être donnée au curé à la fin de l'été. ${ }^{97}$

Mgr Dosquet ira même jusqu'à refuser l'absolution et les sacrements aux maîtres qui enseigneront à des personnes de différent sexe. ${ }^{98}$ Les curés sont obligés de refuser l'absolution aux cabaretiers et à ceux qui « gagnent leur vie par ce détestable commerce ».99 Dans un Mandement contre la traite des boissons aux sauvages, il déplore «la grossièreté de ce crime» et le considère comme un «torrent d'iniquité qui grossit de jour en jour et coule avec plus de rapidité que jamais ». A ces causes, il défend à tous les confesseurs du diocèse d'absoudre ceux qui, directement ou indirectement, contribuent à enivrer les sauvages, se réservant à lui seul le pouvoir d'absoudre de ce erime. ${ }^{1}$ En France, Colbert, se plaint à Bellinzani de la même rigueur et lui dit qu'il ne peut recevoir l'absolution ni faire ses Pâques, parce qu'il professe une opinion différente du clergé du Canada et des Docteurs de la Sorbonne sur la question de l'eau-de-vie. ${ }^{2}$ Les officiers qui retiennent la solde de leurs soldats se verront aussi privés du sacrement de pénitence, s'ils ne consentent à réparer leurs torts. Ainsi, Vaudreuil, le gouverneur, et plusieurs officiers du temps sont privés des sacrements. ${ }^{3}$ Dans la Vie de $M g r$ de St-Vallier, on signale deux exemples célèbres et com-

95 Mandements, I: 279.

96 Ibid., 279, 491s.

${ }^{97}$ Ivanhoë Caron, Inventaire des documents concernant l'Eglise du Canada, sous le régime français, dans RAPQ, pour l'année 1940-1941, 356.

98 Gosselin, L'Eglise du Canada, IIe partie, 289; Mandements, I: 546.

${ }^{99}$ Mandements, I: $511 \mathrm{~s}$.

1 Mandement du 26 nov. 1730. Mandements, I: 536.

2 Gosselin, Vie de Mgr de St-Vallier, I: 123, et Mandements, 1: 189s., 312s., 334, 347ss.

3 Gosselin, Vie de Mgr de Laval, II: 191. 
pliqués - parce qu'ils ont fait du bruit - de personnes à qui on avait refusé l'absolution et les billets de confession. ${ }^{4}$

\section{L'Interdit.}

Etaient frappés d'Interdit les gens qui contrevenaient à des lois importantes de l'Église. L'exemple le plus frappant, où l'on voit l'application de ces foudres, est celui que fit $\mathrm{Mgr}$ de St-Vallier contre les Récollets de Montréal. Ceux-ci, lors de l'inauguration de leur Église et Couvent, avaient invité $\mathrm{Mgr}$ de St-Vallier, M. de Callières, gouverneur de la ville, M. de Champigny, intendant, et d'autres dignitaires. A la cérémonie du matin, survint l'affaire du prie-Dieu ${ }^{5}$. Au banquet qui suivit, s'amène tout à coup un cortège de femmes, besace au dos; elles font le tour des tables à plusieurs reprises, quêtant des mets, pour enfin s'en aller. Parmi ces dames, il y en avait une, Mme de Ramsay, sœur du P. Denis, gardien, dont la présence convenait encore moins dans ce lieu que celle des autres. Après le refus des Pères d'ôter tous les prie-Dieu de l'église, comme il l'avait demandé, $\mathrm{Mgr}$ décide d'interdire l'église et il le signifie par lettre, datée du 13 mai 1694.

Le P. Perreault, récollet de Québec, essaie d'arranger les choses; M. de Champigny lui-même s'efforce de raccommoder

${ }^{4}$ Gosselin, Vie de Mgr de St-Vallier, I: 340s. - Dans le premier cas, il s'agit d'un nommé Garnault et de sa femme qui sont tous deux renvoyés par le Conseil devant l'official, en 1712. Jugements du Conseil Supérieur, VI: 489; dans le second cas 1714, il s'agit de Pierre Le Boullenger de StPierre et Marie-Renée Godefroy son épouse, qui présentant une requête au Conseil, au nom et comme prenant fait et cause d'Anne-Marguerite Le Boullenger, leur fille. Gosselin, Vie de Mgr de St-Vallier, I: $340 \mathrm{~s}$.

5 Callières. Il s'agit de Louis-Hector, chevalier de Callières (16461703), lieutenant, capitaine, gouverneur de Montréal et de la NouvelleFrance. Il venait de recevoir la Croix de Chevalier de St-Louis, lorsque éclata, à Ville-Marie, l'incident du PRIE-DIEU, à l'église des Récollets, que l'on inaugurait. Mgr de St-Vallier, avait le sien du côté droit et le gouverneur avait le sien au milieu; cette disposition donna lieu à toute une scène! Mgr de St-Vallier donna ordre au Prieur de lui céder les honneurs de la préséance, tandis que $M$. de Callières, s'obstina à se l'attribuer. Le Prélat lança l'interdit sur l'église et le couvent. M. de Champigny, intendant, affirma, le 17 oct., au Ministre, que $M$. de Callières était désigné dans l'interdiction, à cause d'un commerce prolongé qu'il entretenait avec la sour du Prieur des Récollets. Cf. LeJeune, Dictionnaire général (...) $d u$ Canada, à l'art. Callières, 276-279. 
M. de Callières avec l'Évêque, pendant que Frontenac fait tout pour fléchir le prélat. Bien plus, le Discrétoire des Pères se réunit à Québec, le 6 juillet, et il envoie une protestation à l'Èvêque, qui ne cède pas. Alors, ils ouvrent leur église. L'Évêque envoie, coup sur coup, trois monitions et donne les raisons de sa conduite: liaison d'intérêt du Supérieur avec M. de Callières, entrée libre des femmes dans le Monastère. ${ }^{6}$ Puis, il prononce de nouveau l'interdit contre tous les Récollets de Montréal. De nouveau, leur Discrétoire de Québec déclare l'interdit nul dans le fond et dans la forme, et ordonne à tous les religieux de continuer leurs fonctions. M. de Callières proteste par des affichages tapageurs à la porte de l'église. Monseigneur répond qu'il passe justement en France pour y porter le cas au roi et obtenir justice et réparation. ${ }^{7}$

Cet incident regrettable rappelle une autre querelle survenue entre l'autorité épiscopale et ces mêmes Récollets. Ceux-ci avaient obtenu, par un permis du Roi, en date du 27 octobre 1681, l'autorisation d'ouvrir une infirmerie à la haute-ville de Québec. Ils pouvaient y célébrer la messe, portes closes, en faveur de leurs religieux malades, jusqu'à ce qu'ils soient en état de se rendre au Couvent Notre-Dame-des-Anges. Or, cette infirmerie devint bientôt un véritable Hospice, puis un Couvent; l'autel devint une église. Les portes s'ouvrirent; les pénitents y vinrent et le public y fut reçu. On prêchait, confessait, donnait la communion, faisait le catéchisme aux enfants, que l'on prenait même comme pensionnaires. Bientôt on y éleva un clocher ! Malgré les défenses formelles de l'Évêque, les Récollets se réclamaient des privilèges de Réguliers exempts. Un des leurs venant à mourir, $\mathrm{Mgr}$ de Laval refuse qu'on l'y enterre; mais ils passent outre. M. de La Barre écrit au Ministre que c'est une mauvaise affaire que tout cela ! Monseigneur doit finalement leur interdire toutes fonctions ecclésiastiques, et même il écrit au roi pour qu'il intervienne. Celui-ci commence par faire

6 Voir l'opinion de Mgr Dosquet sur cet abus qu'il avait remarqué, dès son arrivée au pays. Gosselin, Vie de Mgr de St-Vallier, II: 93s, I: 121.

7 Gosselin, Vie de Mgr de St-Vallier, I: 117-121. 
abattre le clocher. L'affaire traîna en longueur et chacun resta sur ses positions. ${ }^{8}$

Encore du temps de Mgr de Laval, deux époux de la côte de Lauzon, Jean Dumets et sa femme, ayant refusé de faire baptiser leur enfant, Monseigneur dut les sommer de le faire baptiser avant huit jours, sinon il porterait contre eux l'Interdit de l'Église et les refuserait à la participation aux sacrements. ${ }^{9}$

Après le scandale produit par l'inhumation irrégulière et clandestine des restes mortels de Mgr de St-Vallier, le Vicaire général Boullard dut interdir solennellement et la chapelle de l'Hôpital Général et la Supérieure de l'Institution. ${ }^{10}$

$$
\text { (à suivre) }
$$

Sherbrooke, Québec.

\section{VALÉRIEN ROY}

8 Gosselin, Vie de Mgr de Laval, II : 95-104.

- Ordonnances contre Jean Dumets sur le refus de baptiser son enfant; le 21 oct. 1681, dans Mandements, I: 104s; Gosselin, op. cit., II: 44.

10 Mandements, I: $522 \mathrm{~s}$. 